\title{
NEW CLASSES OF $k$-UNIFORMLY CONVEX AND STARLIKE FUNCTIONS
}

\author{
ESSAM AQLAN, JAY M. JAHANGIRI AND S. R. KULKARNI
}

\begin{abstract}
Certain classes of analytic functions are defined which will generalize new, as well as well-known, classes of k-uniformly convex and starlike functions. We provide necessary and sufficent coefficient conditions, distortion bounds, extreme points and radius of starlikeness for these classes.
\end{abstract}

\section{Introduction}

Let $\mathcal{A}$ denote the family of functions $f$ that are analytic in the open unit disc $\Delta=\{z$ : $|z|<1\}$ and consider the subclass $\mathcal{T}$ consisting of functions $f$ in $\mathcal{A}$ which are univalent in $\Delta$ and are of the form $f(z)=z-\sum_{m=2}^{\infty} a_{m} z^{m}$ where $a_{m} \geq 0$. The class $\mathcal{T}$ was introduced and studied by Silverman [9]. For $0 \leq \lambda \leq 1,0 \leq \beta<1$ and $k \geq 0$ we let $\mathcal{U}(k, \beta, \lambda)$ consist of functions $f$ in $\mathcal{T}$ satisfying the condition

$$
\operatorname{Re}\left(\frac{z f^{\prime}(z)+\lambda z^{2} f^{\prime \prime}(z)}{(1-\lambda) f(z)+\lambda z f^{\prime}(z)}\right) \geq k\left|\frac{z f^{\prime}(z)+\lambda z^{2} f^{\prime \prime}(z)}{(1-\lambda) f(z)+\lambda z f^{\prime}(z)}-1\right|+\beta .
$$

The family $\mathcal{U}(k, \beta, \lambda)$ is of special interest for it contains many well-known, as well as new, classes of analytic univalent functions. In particular, $\mathcal{U}(0, \beta, 0)$ is the family of functions starlike of order $\beta$ and $\mathcal{U}(0, \beta, 1)$ is the family of functions convex of order $\beta$. For $\mathcal{U}(k, 0,0)$ and $\mathcal{U}(k, 0,1)$ we, respectively, obtain the classes of k- uniformly starlike and k-uniformly convex functions. The case for $\beta$ to be other than zero, i.e. $\beta \in(0,1)$, is of special interest. For instance, if $\beta \in(0,1)$ then these classes are generalized to $\mathcal{U}(k, \beta, 0)$ and $\mathcal{U}(k, \beta, 1)$ of $\mathrm{k}$-uniformly starlike functions of order $\beta$ and k-uniformly convex functions of order $\beta$. More generally speaking, as $\beta$ and $\lambda$ vary, the family $\mathcal{U}(k, \beta, \lambda)$ provides a transition from the class k-uniformly starlike functions of order $\beta$ and type $\lambda$ to the class of k-uniformly convex functions of order $\beta$ and type $\lambda$ in $\Delta$. The main feature of the elements of these classes is the fact that they map circular arcs with center at any point $\zeta$ in the open unit disk $\Delta$ onto convex arcs or arcs starlike with respect to $f(\zeta)$, respectively. We remark that the classes of uniformly convex and uniformly starlike functions were introduced by Goodman [3,4] and later generalized by Kanas et

Received April 11, 2001; revised September 10, 2001.

2000 Mathematics Subject Classification. Primary 30C45, secondary 30C50.

Key words and phrases. Analytic, uniformly convex, starlike. 
al. in $[5,6,7,8]$. In this paper we provide necessary and sufficent coefficient conditions, distortion bounds, extreme points, radius of starlikeness and convexity, closure theorem for functions in $\mathcal{U}(k, \beta, \lambda)$.

\section{Main Results}

Our first theorem is on the necessary and sufficient coefficient requirements for functions to be in the class $\mathcal{U}(k, \beta, \lambda)$.

Theorem 1. $f \in \mathcal{U}(k, \beta, \lambda)$ if and only if

$$
\sum_{m=2}^{\infty}(1+m \lambda-\lambda)(m(1+k)-(k+\beta)) a_{m} \leq 1-\beta
$$

where $0 \leq \beta<1, k \geq 2,0 \leq \lambda \leq 1$, and $-\pi<\theta \leq \pi$.

Proof. We have $f \in \mathcal{U}(k, \beta, \lambda)$ if and only if the condition (1.1) is satisfied. Upon using the fact that

$$
\operatorname{Re} \quad w>k|w-1|+\beta \Leftrightarrow \operatorname{Re}\left\{w\left(1+k e^{i \theta}\right)-k e^{i \theta}\right\}>\beta
$$

the condition (1.1) may be written as

$$
\operatorname{Re}\left(\frac{z f^{\prime}(z)+\lambda z^{2} f^{\prime \prime}(z)}{(1-\lambda) f(z)+\lambda z f^{\prime}(z)}\left(1+k e^{i \theta}\right)-k e^{i \theta}\right) \geq \beta
$$

or equivalenlty

$$
\operatorname{Re}\left(\frac{\left(z f^{\prime}(z)+\lambda z^{2} f^{\prime \prime}(z)\right)\left(1+k e^{i \theta}\right)-k e^{i \theta}\left((1-\lambda) f(z)+\lambda z f^{\prime}(z)\right)}{(1-\lambda) f(z)+\lambda z f^{\prime}(z)}\right) \geq \beta .
$$

Now we let $A(z)=\left[z f^{\prime}(z)+\lambda z^{2} f^{\prime \prime}(z)\right]\left(1+k e^{i \theta}\right)-k e^{i \theta}\left[(1-\lambda) f(z)+\lambda z f^{\prime}(z)\right]$ and let $B(z)=(1-\lambda) f(z)+\lambda z f^{\prime}(z)$. Then $(2.2)$ is equivalent to

$$
|A(z)+(1-\beta) B(z)| \geq|A(z)-(1+\beta) B(z)| \text { for } 0 \leq \beta<1 .
$$

For $A(z)$ and $B(z)$ as above, we have

$$
\begin{aligned}
|A(z)+(1-\beta) B(z)|= & \mid(2-\beta) z-\sum_{m=2}^{\infty}(m+m \lambda(m-1)+(1-\beta)(1-\lambda+m \lambda)) a_{m} z^{m} \\
& -k e^{i \theta}\left(\sum _ { m = 2 } ^ { \infty } \left[(m+m \lambda(m-1)-(1-\lambda+m \lambda)] a_{m} z^{m} \mid\right.\right. \\
\geq & (2-\beta)|z|-\sum_{m=2}^{\infty}[m+m \lambda(m-1)+(1-\beta)(1-\lambda+m \lambda)] a_{m}|z|^{m} \\
& -k \sum_{m=2}^{\infty}[m+m \lambda(m-2)-1+\lambda] a_{m}|z|^{m}
\end{aligned}
$$


and, similarly,

$$
\begin{gathered}
|A(z)-(1+\beta) B(z)| \leq \beta|z|+\sum_{m=2}^{\infty}[m+m \lambda(m-1)-(1+\beta)(1-\lambda+m \lambda)] a_{m}|z|^{m} \\
+k \sum_{m=2}^{\infty}[m+m \lambda(m-1)-(1-\lambda+m \lambda)] a_{m}|z|^{m} .
\end{gathered}
$$

Therefore,

$$
\begin{aligned}
& |A(z)+(1-\beta) B(z)|-|A(z)-(1+\beta) B(z)| \\
\geq & 2(1-\beta)|z|-\sum_{m=2}^{\infty}[2 m+2 m \lambda(m-1)-2 \beta(1-\lambda+m \lambda)] \\
& -k[2 m+2 m \lambda(m-1)-2(1-\lambda+m \lambda)] a_{m}|z|^{m} \geq 0
\end{aligned}
$$

or

$$
(1-\beta) \geq \sum_{m=2}^{\infty}[m(1+k)+m \lambda(m-1)(1+k)-(1-\lambda+m \lambda)(\beta+k)] a_{m}
$$

which yields

$$
(1-\beta) \geq \sum_{m=2}^{\infty}(1-\lambda+m \lambda)(m(1+k)-(k+\beta)] a_{m} .
$$

On the other hand, we must have

$$
\operatorname{Re}\left(\frac{\left[z f^{\prime}(z)+\lambda z^{2} f^{\prime \prime}(z)\right]\left(1+k e^{i \theta}\right)-k e^{i \theta}\left[(1-\lambda) f(z)+\lambda z f^{\prime}(z)\right]}{(1-\lambda) f(z)+\lambda z f^{\prime}(z)}\right) \geq \beta .
$$

Upon choosing the values of $z$ on the positive real axis where $0 \leq z=r<1$, the above inequality reduces to

$$
\begin{array}{r}
\operatorname{Re}\left((1-\beta)-\sum_{m=2}^{\infty}\left[m-m^{2} \lambda-\lambda-\beta(1-\lambda+m \lambda)\right] a_{m} r^{m-1}\right. \\
\left.-k e^{i \theta} \sum_{m=2}^{\infty}\left[m+m^{2} \lambda-\lambda-(1-\lambda+m \lambda)\right] a_{m} r^{m-1}\right) \\
\left./\left(1-\sum_{m=2}^{\infty}(1-\lambda+m \lambda) a_{m} r^{m-1}\right)\right) \geq 0 .
\end{array}
$$

Since $\operatorname{Re}\left(-e^{i \theta}\right) \geq-\left|e^{i \theta}\right|=-1$, the above inequality reduces to

$$
\operatorname{Re}\left(\left((1-\beta)-\sum_{m=2}^{\infty}\left[m+m^{2} \lambda-\lambda-\beta(1-\lambda+m \lambda)\right] a_{m} r^{m-1}\right.\right.
$$




$$
\begin{aligned}
\left.-k \sum_{m=2}^{\infty}\left[m+m^{2} \lambda-\lambda-(1-\lambda+m \lambda)\right] a_{m} r^{m-1}\right) & \\
\left./\left(1-\sum_{m=2}^{\infty}(1-\lambda+m \lambda) a_{m} r^{m-1}\right)\right) & \geq 0 .
\end{aligned}
$$

Letting $r \rightarrow 1^{-}$we get desired conclusion.

Remark. As special cases of Theorem 1, for $\lambda=0$, see [2] and for $k=0$, see [1].

The distortion theorem for the class $\mathcal{U}(k, \beta, \lambda)$ is given next.

Theorem 2. If $f \in \mathcal{U}(k, \beta, \lambda)$ and $|z| \leq r<1$, then we have the sharp bounds

$$
r-\frac{1-\beta}{(1+\lambda)(2+k-\beta)} r^{2} \leq|f(z)| \leq r+\frac{1-\beta}{(1+\lambda)(2+k-\beta)} r^{2}
$$

and

$$
1-\frac{2(1-\beta)}{(1+\lambda)(2+k-\beta)} r \leq\left|f^{\prime}(z)\right| \leq 1+\frac{2(1-\beta)}{(1+\lambda)(2+k-\beta)} r .
$$

Proof. We only prove the right hand side inequality in (2.3) since the other inequalities can be justified using similar arguments. On account of (2.1), we may write

$$
\sum_{m=2}^{\infty}(1+\lambda)(2+k-\beta) a_{m} \leq \sum_{m=2}^{\infty}(1+m \lambda-\lambda)(m(1+k)-(k+\beta)) a_{m} \leq 1-\beta .
$$

Hence

$$
|f(z)| \leq|z|+|z|^{2} \sum_{m=2}^{\infty} a_{m} \leq r+r^{2} \sum_{m=2}^{\infty} a_{m} \leq r+\frac{1-\beta}{(1+\lambda)(2+k-\beta)} r^{2} .
$$

The distortion bounds in Theorem 2 are sharp for

$$
f(z)=z-\frac{(1-\beta)}{(1+\lambda)(2+k-\beta)} z^{2}, z= \pm r .
$$

In the following theorem, we study the properties of extreme points of functions in the family $\mathcal{U}(k, \beta, \lambda)$.

Theorem 3. Let $f_{1}(z)=z$ and $f_{m}(z)=z-\frac{1-\beta}{(1+m \lambda-\lambda)(m(1+k)-(k+\beta))} z^{m}$ where $\lambda \geq 0$, $0 \leq \beta<1, k \geq 0$, and $m \geq 2$. Then $f(z)$ is in $\mathcal{U}(k, \beta, \lambda)$ if and only if it can be expressed in the form $f(z)=\sum_{m=1}^{\infty} \gamma_{m} f_{m}(z)$ where $\gamma_{m} \geq 0$ and $\sum_{m=1}^{\infty} \gamma_{m}=1$.

Proof. Let $f(z)=\sum_{m=1}^{\infty} \gamma_{m} f_{m}(z)$ where $\gamma_{m} \geq 0$ and $\sum_{m=1}^{\infty} \gamma_{m}=1$. Letting

$$
f(z)=z-\sum_{m=2}^{\infty} \frac{1-\beta}{(1+m \lambda-\lambda)(m(1+k)-(k+\beta))} \gamma_{m} z^{m} .
$$


we get

$$
\begin{aligned}
& \sum_{m=2}^{\infty}\left(\frac{(1+m \lambda-\lambda)(m(1+k)-(k+\beta))}{1-\beta}\right) \gamma_{m} \frac{1-\beta}{(1+m \lambda-\lambda)(m(1+k)-(k+\beta))} \\
= & \sum_{m=2} \gamma_{m}=1-\gamma_{1} \leq 1 \quad \text { (by Theorem 1). }
\end{aligned}
$$

Therefore $f \in \mathcal{U}(k, \beta, \lambda)$. Conversely, suppose that $f \in \mathcal{U}(k, \beta, \lambda)$. Then

$$
a_{m} \leq \frac{1-\beta}{(1+m \lambda-\lambda)(m(1+k)-(k+\beta))}, \quad(m \geq 2) .
$$

Now, by letting $\gamma_{m}=\frac{(1+m \lambda-\lambda)(m(1+k)-(k+\beta))}{1-\beta} a_{m}$ and $\gamma_{1}=1-\sum_{m=2}^{\infty} \gamma_{m}$ we conclude the theorem, since $f(z)=\sum_{m=1}^{\infty} \gamma_{m} f_{m}=\gamma_{1} f_{1}(z)+\sum_{m=2}^{\infty} \gamma_{m} f_{m}(z)$.

Remark. For $\lambda=0$, we obtain the extreme points given earlier in [2].

Finally, we discuss the radius of starlikeness of the functions in $\mathcal{U}(k, \beta, \lambda)$.

Theorem 5. Let the $f$ be in the class $\mathcal{U}(k, \beta, \lambda)$. Then $f$ is starlike of order $\delta(0 \leq$ $\delta<1)$ in $|z|<r_{2}(\beta, \lambda, k, \delta)$, where

$$
r_{2}(\beta, \lambda, k, \delta)=\inf _{m}\left(\frac{(1-\delta)(1+m \lambda-\lambda)(m(1+k)-(k+\beta))}{(m-\delta)(1-\beta)}\right)^{\frac{1}{m-1}}, \quad m \geq 2 .
$$

Proof. It suffices to show that $\left|\frac{z f^{\prime}(z)}{f(z)}-1\right| \leq 1-\delta$ for $|z|<r_{2}(\beta, \lambda, k, \delta)$. Note that

$$
\left|\frac{z f^{\prime}(z)}{f(z)}-1\right| \leq \frac{\sum_{m}^{\infty}(m-1) a_{m}|z|^{m-1}}{1-\sum_{m}^{\infty} a_{m}|z|^{m-1}}
$$

Now $\left|\frac{z f^{\prime}(z)}{f(z)}-1\right| \leq 1-\delta$ if we have the condition

$$
\sum_{m=2}^{\infty} \frac{(m-\delta) a_{m}|z|^{m-1}}{(1-\delta)} \leq 1
$$

Considering the coefficient conditions required by Theorem 1 , the above inequality (2.5) is true if

$$
\frac{m-\delta}{1-\delta}|z|^{m-1} \leq \frac{(1+m \lambda-\lambda)(m(1+k)-(k+\beta))}{(1-\beta)}
$$

or if

$$
|z| \leq\left\{\frac{(1-\delta)(1+m \lambda-\lambda)(m(1+k)-(k+\beta))}{(m-\delta)(1-\beta)}\right\}^{\frac{1}{m-1}}, \quad m \geq 2 .
$$

This last expression yields the bound required by the above theorem. 


\section{References}

[1] O. Altintas, On a subclass of certain starlike functions with negative coefficient, Math. Japon. 36 (1991), 489-495.

[2] R. Bharati, R. Parvatham and A. Swaminathan, On subclasses of uniformaly convex functions and correspondding class of starlike functions, Tamkang J. Math. 28 (1997), 17-32.

[3] A. W. Goodman, On uniformly convex functions, Ann. Polon. Math. 56 (1991), 87-92.

[4] A. W. Goodman, On uniformly starlike functions, J. Math. Anal. Appl. 155 (1991), 364-370.

[5] S. Kanas and A. Wisniownska, Conic regions and k-uniform convexity, J. Comput. Appl. Math. 105 (1999), 327-336.

[6] S. Kanas and A. Wisniownska, Conic region and k-uniform convexity, II, Zeszyty. Nauk. Politech. Rzes. Mat. 22 (1998), 65-78.

[7] S. Kanas and A. Wisniownska, Conic domains and starlike functions, Rev. Roumaine Math. Pures Appl. 45 (2000), 647-657.

[8] S. Kanas and H. M. Srivastava, Linear operators associated with k-uniformly convex functions, Integral Transform Spec. Funct. 9 (2000), 121-132.

[9] H. Silverman, Univalent functions with negative coefficients, Proc. Amer. Math. Soc. 51 (1975), 109-116.

Department of Mathematics, Fergusson College, Pune - 411 004, India.

Mathematical Sciences, Kent State University, 14111 Claridon Troy Road, Burton, Ohio 440219500, U.S.A.

E-mail: jay@geauga.kent.edu

Department of Mathematics, Fergusson College, Pune - 411 004, India.

E-mail: Kulkarni_ferg@yahoo.com 